\title{
Documents et espaces de communication numériques dans un environnement support de projets entre apprenants
}

\author{
Sébastien George****1 - Pascal Leroux* \\ * Laboratoire d'Informatique de l'Université du Maine (LIUM) \\ Avenue René Laënnec - 72085 Le Mans Cedex 9 - France \\ \{george,leroux\}@lium.univ-lemans.fr \\ ** Centre de recherche LICEF-Télé-université du Québec \\ 4750 avenue Henri-Julien, Montréal, Qc. H2T 3E4 - Canada \\ sgeorge@licef.teluq.uquebec.ca
}

\begin{abstract}
RÉSUMÉ. Les documents numériques et les espaces numériques de communication tiennent une place centrale dans les environnements CSCL (Computer Supported Collaborative Learning). Ils permettent l'échange d'informations et le partage de connaissances entre apprenants. Dans cet article, nous les abordons selon deux points de vue. Le premier porte sur l'utilisation de documents structurés par des gabarits pour faciliter la rédaction et l'exploitation de documents par les apprenants. Le second point de vue concerne l'analyse d'espaces numériques de communication semi-structurée dans le but de déterminer des profils de comportements sociaux d'apprenants.

ABSTRACT. Electronic documents and electronic communication spaces hold a key position in CSCL (Computer Supported Collaborative Learning) environments. They allow information exchange and knowledge sharing between learners. In this paper, we present two approaches to tackle them. The first one deals with the use of structured documents by templates to make them easier to write and read. The second approach concerns automatic analysis of semistructured communication in order to determine social behavior profiles of learners.

MOTS-CLÉS: formation à distance, CSCL, gabarits de documents, analyse automatique de conversations médiatisées, profils de comportementaux sociaux

KEYWORDS: distance education, CSCL, document templates, automatic analysis of computermediated conversations, social behavior profiles
\end{abstract}

\footnotetext{
${ }^{1}$ Le premier auteur a réalisé les travaux de recherche présentés dans cet article dans le cadre de son travail de thèse au LIUM et effectue actuellement un post-doctorat au centre de recherche LICEF de la Télé-université du Québec à Montréal.
}

Revue Document Numérique 


\section{Introduction}

Le contexte général de notre travail concerne les environnements informatiques pour l'apprentissage à distance et plus précisément les environnements visant à favoriser l'apprentissage collectif, c'est-à-dire se fondant sur une approche pédagogique impliquant les apprenants dans des activités collectives et les incitant ainsi à partager leurs connaissances dans le but de favoriser la construction sociale des savoirs. Nous nous situons donc dans le domaine de recherche des CSCL (Computer-Supported Collaborative Learning) [BAN 89].

Les documents numériques et les espaces numériques de communication tiennent une place centrale dans les environnements CSCL. Ils permettent les échanges d'informations et la capitalisation des connaissances entre apprenants. Dans cet article, nous les abordons selon deux points de vue. Le premier porte sur l'utilisation de documents structurés par des gabarits pour faciliter la rédaction et l'exploitation de documents par les apprenants. Le second point de vue concerne l'analyse d'espaces numériques de communication semi-structurée dans le but de déterminer des profils de comportements sociaux d'apprenants.

Pour nos travaux de recherche, nous avons conçu et développé un environnement CSCL support d'une pédagogie de projet. Dans la première section de l'article, nous décrivons le modèle support d'une pédagogie de projet à distance élaboré sous un angle organisationnel, structurel, informationnel en montrant notamment la place centrale qu'occupent les documents numériques dans ce modèle. Nous présentons ensuite, dans la section 3, l'environnement informatique support de projets entre apprenants. Dans la section 4, nous développons un aspect de nos travaux concernant l'analyse automatique de conversations textuelles dans l'optique de déterminer des profils de comportements sociaux. Nous terminons par la présentation de résultats d'expérimentations de l'environnement conçu.

\section{Un modèle support d'une pédagogie de projet à distance}

Notre travail repose sur un modèle support d'une pédagogie de projet dans un contexte de distance. Nous présentons dans cette section ce modèle fondé sur une organisation humaine, une structure temporelle et un modèle d'échanges d'informations avant de le décrire dans son ensemble.

\subsection{Modèle organisationnel d'un projet}

Nous structurons l'organisation humaine des projets autour d'un chef de projet, joué par un formateur, et d'une équipe. Nous appelons «équipe » un ensemble d'apprenants qui se trouvent à distance et engagés dans un même projet. Une équipe composée de trois co-équipiers semble être la bonne taille pour des projets. En effet, 
des études sociologiques ont montré que les triades étaient des formes sociales particulièrement intéressantes. D'après Simmel [SIM 02], « il n'y a pas de triade où un désaccord entre deux de ses éléments ne se produise de temps à autre... et où le troisième est donc appelé à jouer le rôle d'arbitre ». Dans ce sens, les travaux de Caplow [CAP 68] ont montré que les triades avaient un effet catalyseur et que les relations binaires étaient modifiées par la présence du troisième élément. Nous choisissons donc de prendre le schéma triadique comme modèle et de composer des équipes de trois apprenants. Quant au chef de projet, son rôle est de définir le projet, de composer l'équipe, de suivre l'évolution du travail et d'assister les apprenants. Il doit être un facilitateur et un consultant. Les sujets apprenants et le chef de projet utilisent des ordinateurs reliés par un réseau informatique pour communiquer et travailler collectivement.

\subsection{Modèle structurel d'un projet}

Parmi les principes d'une méthodologie de projet définie par Goguelin [GOG 94], nous pouvons retenir le principe d'ordre : « un projet doit s'inscrire dans le temps et se décomposer en étapes successives qui forment un schéma prévisionnel d'actions ». La planification des actions est nécessaire pour donner un cadre temporel au projet. Par ailleurs, nous croyons qu'il est bénéfique de structurer les activités collectives et ce pour deux raisons. Premièrement, le fait de fournir une certaine structure permet d'assurer une cohérence et une coordination au niveau des travaux effectués par les apprenants. Deuxièmement, même s'il est vrai qu'un des avantages de l'apprentissage à distance est de donner une certaine forme de liberté et de souplesse dans la manière d'apprendre, cette liberté peut ne pas convenir à certains apprenants qui se sentent un peu perdus et qui peuvent avoir des difficultés à être complètement autonomes [POR 98]. Une structuration de certaines activités peut alors être bénéfique. Bien sûr, il ne faut pas tomber dans l'excès inverse en guidant le projet d'une manière excessive et autoritaire. Néanmoins, le fait de le cadrer a aussi l'avantage de donner un certain rythme aux activités des apprenants.

Par ailleurs, nous nous sommes posé la question de savoir si les activités les plus favorables à l'apprentissage étaient les activités de type synchrone ou asynchrone. Une étude d'utilisation d'une pédagogie de projet dans un contexte de distance a montré l'importance des phases synchrones même si dans ce cas elles se faisaient en face à face [DIR 94]. Cependant, nous pensons qu'une activité collective ponctuelle et synchrone entre apprenants ne permet pas d'établir une dynamique de groupe suffisante. Dans ce sens, Cerratto [CER 99] montre qu'un environnement ne permettant qu'une modalité d'interaction synchrone ne favorise pas le travail de groupe et, au contraire, peut être source de dysfonctionnement (fragmentation de l'espace commun sans coordination). Nous retenons de ces études qu'il faut viser à supporter les moyens de communication et les modalités d'interaction synchrones et asynchrones pour favoriser le travail collectif. 


\subsection{Modèle informationnel d'un projet}

Les productions écrites sont nécessaires à toute activité de projet. L'écrit permet aux utilisateurs de s'informer mutuellement sur ce qu'ils font ou sur ce qu'ils vont faire. Les documents écrits sont alors des objets de liaison entre les sujets. Fischer [FIS 00] les nomme «boundary objects ». Des chercheurs ont observé le rôle articulatoire que peuvent jouer les documents numériques : «le fichier n'est plus un simple produit de l'activité d'un concepteur et représente aussi un support d'ajustements entre différents acteurs de la conception, c'est-à-dire un outil d'articulation du travail individuel et collectif » [SOU 96]. En outre, l'importance de l'écrit pour l'apprentissage a souvent été soulignée. Le fait d'écrire oblige à réfléchir, à conceptualiser, à synthétiser. Le processus d'écriture nécessite d'expliciter sa pensée. La production écrite exige du scripteur une organisation de ses idées [BÉD 98]. La relecture par l'apprenant de documents écrits par des équipiers va l'aider à développer des compétences particulières comme la critique évaluative.

Nous souhaitons permettre à la fois l'écriture de documents individuels et de documents collectifs. En effet, nous avons retenu de l'étude de Cerratto [CER 99] que, même lors d'activités d'écriture de texte en commun, un espace pour l'écriture individuelle est nécessaire sinon les utilisateurs se délimitent des parties privées dans les documents collectifs. Par ailleurs, lorsque seuls des documents individuels existent, les utilisateurs ne sont pas amenés à collaborer pour intégrer les différentes idées. Notre environnement intègre donc les deux types de documents.

En prenant le point de vue d'un apprenant, nous classons les documents en trois catégories : les documents personnels, les documents de ses équipiers et les documents collectifs qui appartiennent à l'équipe. Les droits d'accès sont alors différents selon le type de document. L'apprenant a la possibilité d'accéder et de modifier ses documents personnels. Il peut définir chacun de ses documents comme privé (seulement accessible par lui) ou comme public (accessible en lecture par ses équipiers). Un sujet apprenant peut donc consulter les documents publics de ses équipiers. Les documents d'équipe sont visibles par tous les co-équipiers mais ne sont modifiables que lors d'une réunion synchrone (pour une modification en collaboration). Nous signalons que tous les documents ne sont pas présents dès le début du projet. Leur apparition se fait au fur et à mesure de l'avancement dans le projet. Enfin, les documents de projet sont fournis sous la forme de documents préformatés avec des gabarits. Les sujets apprenants sont alors guidés dans la rédaction des documents. Pour ces gabarits, nous proposons un format spécial constitué de zones non modifiables par les apprenants (texte défini par le professeur en amont du projet) et de zones modifiables (à remplir par les apprenants lors du projet). La Figure 1 représente un exemple de document avec gabarit. L'intérêt d'un gabarit est, d'un côté, de faciliter la rédaction de documents par l'apprenant en lui fournissant une certaine structure et, d'un autre côté, de rendre les documents homogènes et donc plus faciles à exploiter par les autres apprenants. 


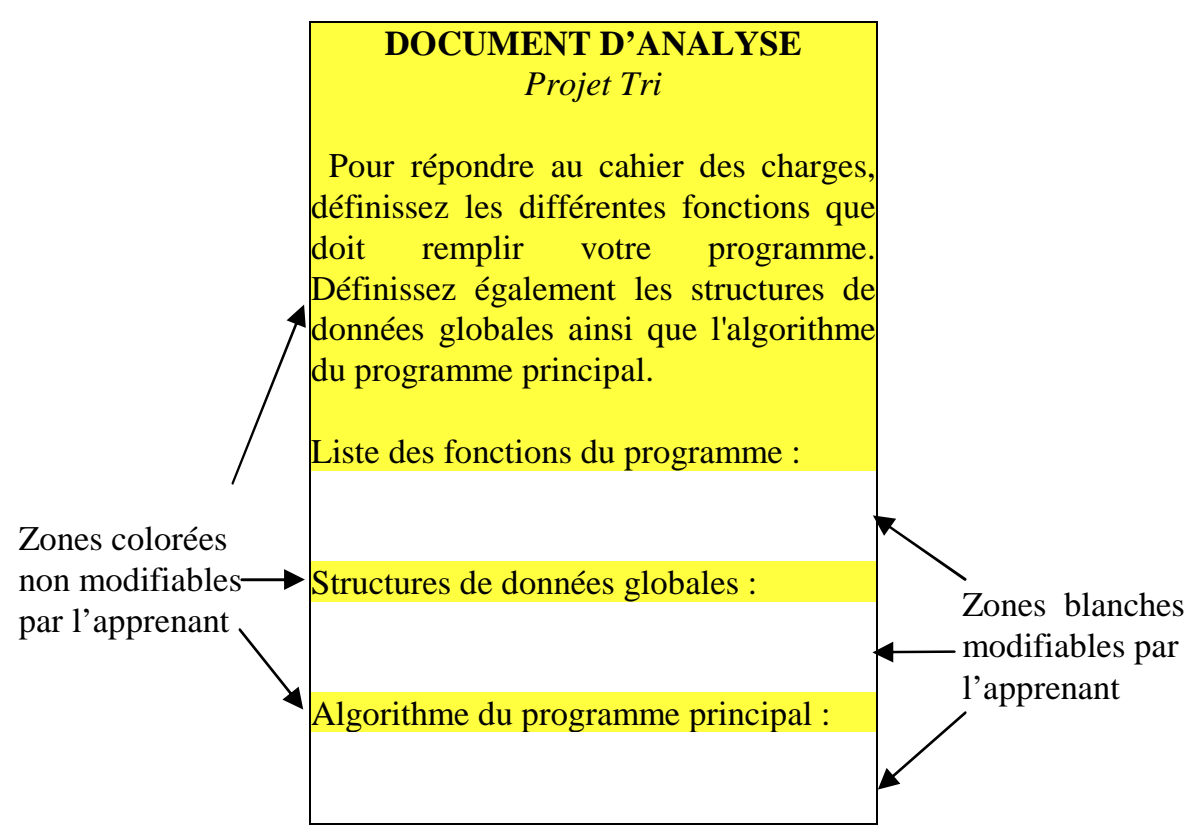

Figure 1. Un exemple de gabarit de document

\subsection{Modèle général d'un projet}

La prise en compte des précédentes considérations nous amène à structurer un projet en plusieurs étapes, chacune d'elles comportant une phase de travail asynchrone puis une phase synchrone (Figure 2). Durant la phase asynchrone, chaque membre de l'équipe effectue une tâche particulière et rédige un document individuel. Durant la phase synchrone, la tâche à effectuer est une tâche d'équipe et un document est rédigé en collaboration.

De ce modèle général ressort l'importance des documents numériques et de leur rôle articulatoire dans l'échange d'informations entre les phases individuelles et collectives d'une étape et entre les étapes. La forte structuration du projet est volontaire dans un contexte pédagogique pour amener les apprenants à apprendre à travailler en équipe. Cependant, ce modèle est également flexible et comporte une certaine souplesse dans sa mise en œuvre en fonction du déroulement du projet. 


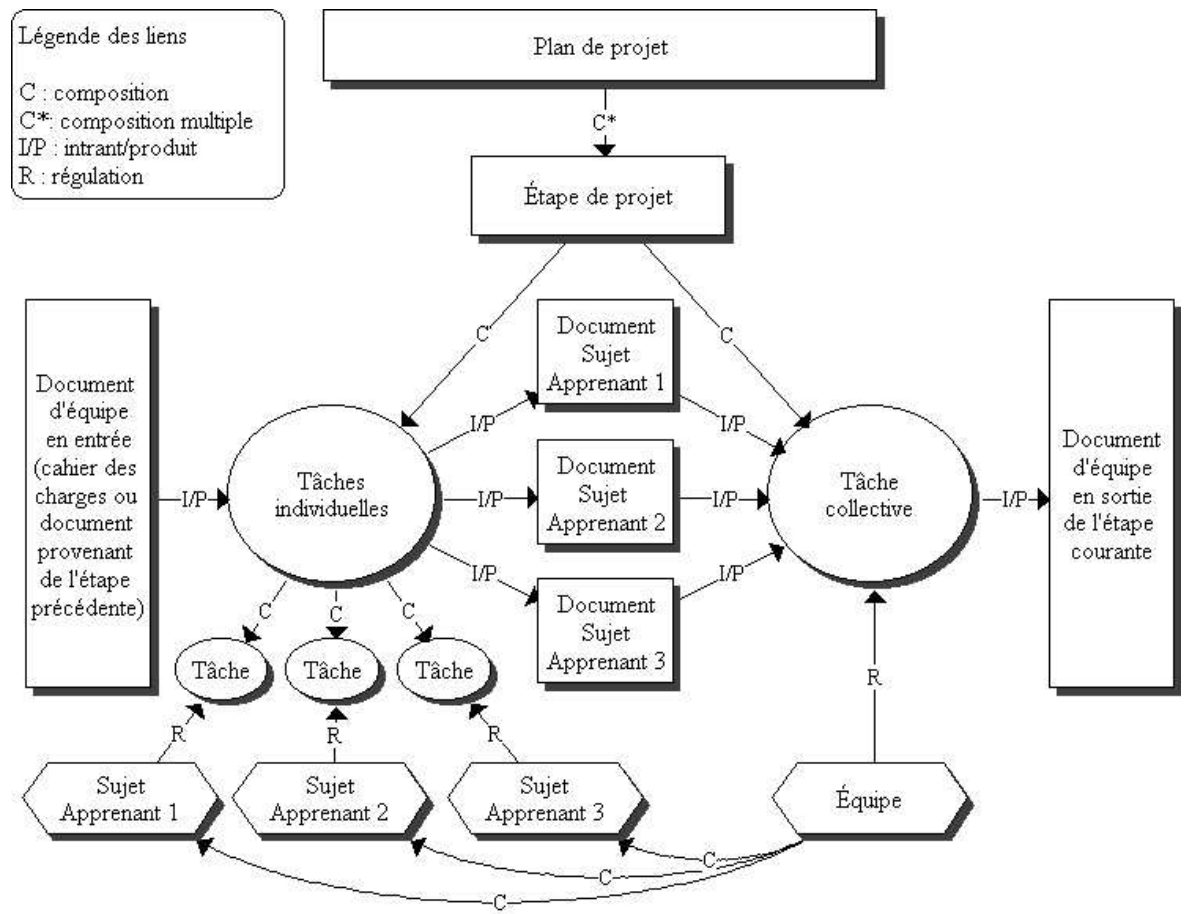

Figure 2. Modèle général d'un projet ${ }^{2}$

\section{Un environnement support d'une pédagogie de projet à distance}

La modélisation générale d'un projet est la base sur laquelle nous nous sommes appuyés pour concevoir et développer un environnement informatique support d'une pédagogie de projet. Cet environnement, nommé SPLACH ${ }^{3}$, repose sur une architecture client/serveur, le développement étant réalisé en java.

\subsection{Présentation globale de l'environnement}

Il y a plusieurs façon d'envisager les moyens technologiques à fournir aux apprenants pour qu'ils puissent réaliser une activité collective à distance. Une des possibilités est qu'ils utilisent différents outils du commerce : par exemple Word ${ }^{\circledR}$

\footnotetext{
${ }^{2}$ Ce modèle a été réalisé en utilisant la technique de modélisation par objets typés (MOT) développée au Centre de recherche LICEF de la Télé-université à Montréal [PAQ 96]. Nous précisons qu'un lien I/P relie une procédure et des données. Ces données sont soit des entrées pour la procédure (Intrant), soit une production de la procédure (Produit).

${ }^{3}$ SPLACH : Support d'une pédagogie de Projet pour l'Apprentissage Collectif Humain.
} 
pour éditer un document, Eudora ${ }^{\circledR}$ pour envoyer du courrier électronique et Netmeeting ${ }^{\circledR}$ pour faire des réunions synchrones. Cette multiplication de logiciels utilisés entraîne une charge cognitive importante pour l'utilisateur qui n'est pas favorable dans un contexte d'apprentissage. Plus simple sera l'interface et l'utilisation des outils, plus les apprenants pourront se concentrer sur leurs activités. Cette simplicité passe par la conception d'un environnement intégrant les outils nécessaires. Certes, l'effort et le temps de développement s'en trouvent considérablement allongés, mais c'est un passage nécessaire pour aboutir à un système exploitable par une majorité d'apprenants.

Par ailleurs, les logiciels existant sont assez hermétiques et ne permettent pas une analyse automatique facile de leur utilisation. Un de nos objectifs à moyen terme est de soutenir au mieux l'apprentissage collectif à partir des informations issues du système informatique (cette partie de notre recherche est décrite dans la section 4). La conception et le développement de nos propres outils nous permettent d'obtenir des traces sur les activités individuelles et collectives, ces traces étant par la suite analysées et exploitées par le système dans le but d'assister les utilisateurs.

L'environnement SPLACH comprend un outil de documentation pour rédiger des comptes-rendus numériques au cours du projet, un outil de planification, un outil de réunion synchrone, des outils de communication asynchrone (courrier électronique et forum de discussion) et enfin des outils spécifiques au domaine d'apprentissage [GEO 01]. En ce qui concerne les outils spécifiques, ils constituent la partie de l'environnement variable d'un domaine d'apprentissage à l'autre. Nous présentons dans la suite de cette section l'outil de support à la documentation et l'outil de support à la co-conception en réunion synchrone.

\subsection{Support à la création et à la gestion de documents}

Dans l'environnement SPLACH, un outil de documentation permet de créer et de gérer les documents d'un projet. L'outil de documentation comprend trois parties (en bas de la Figure 3). Chacune de ces parties contient une liste de documents correspondant à une catégorie précise : la première liste est celle des documents collectifs de l'équipe, la seconde est celle des documents individuels de l'apprenant, et la troisième représente les documents des équipiers. Pour cette troisième liste, des onglets permettent de voir la liste des documents d'un équipier particulier. L'utilisateur peut avec cet outil ouvrir un document parmi ces listes. L'ouverture se fait alors dans l'espace principal de l'environnement (en haut à gauche de la Figure 3). Selon les droits d'accès (propriétaire ou non), le document s'ouvre en mode modification ou en lecture seule. L'utilisateur définit pour chacun de ses documents les propriétés d'accès à celui-ci (public ou privé). Ainsi, chaque participant a sa propre perception et une certaine vue sur les documents du projet. 


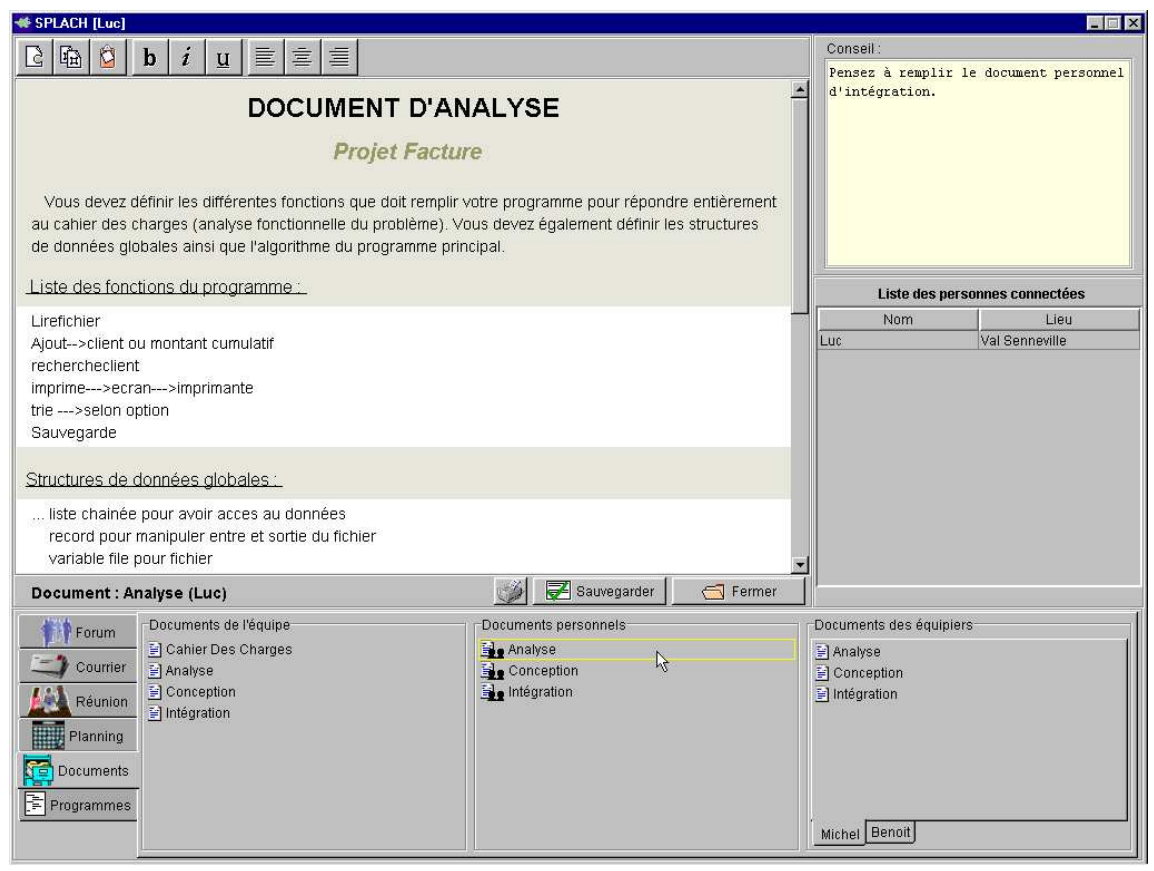

Figure 3. L'outil de documentation

Les documents viennent s'afficher dans les listes au fur et à mesure de l'avancement du projet. Ainsi, en début de projet l'utilisateur ne voit que le cahier des charges (document d'équipe) et, par exemple, un document individuel d'analyse vide mais préformaté par un gabarit (Figure 1). L'objectif est de fournir uniquement les éléments pertinents par rapport à l'étape en cours dans le projet.

\subsection{Support à la co-conception de documents}

L'outil de réunion synchrone (Figure 4) poursuit trois objectifs : permettre le partage et la rédaction en collaboration de documents, soutenir la conversation écrite et assurer une sensation de conscience mutuelle. En ce qui concerne le partage de documents, les apprenants se passent le contrôle sur celui-ci de manière intuitive (lever la main pour demander le contrôle et passer la main pour donner le contrôle au suivant l'ayant demandé). Un des objectifs est de permettre à l'équipe de s'autogérer dans cette collaboration, ce qui contribue selon nous à l'apprentissage du travail en équipe. Pendant une réunion, la conversation entre participants s'effectue par des messages écrits au moyen d'une interface semi-structurée (en bas de l'écran). Un des objectifs de cette interface est de permettre l'analyse automatique de comportements sociaux (cf. section 4). 


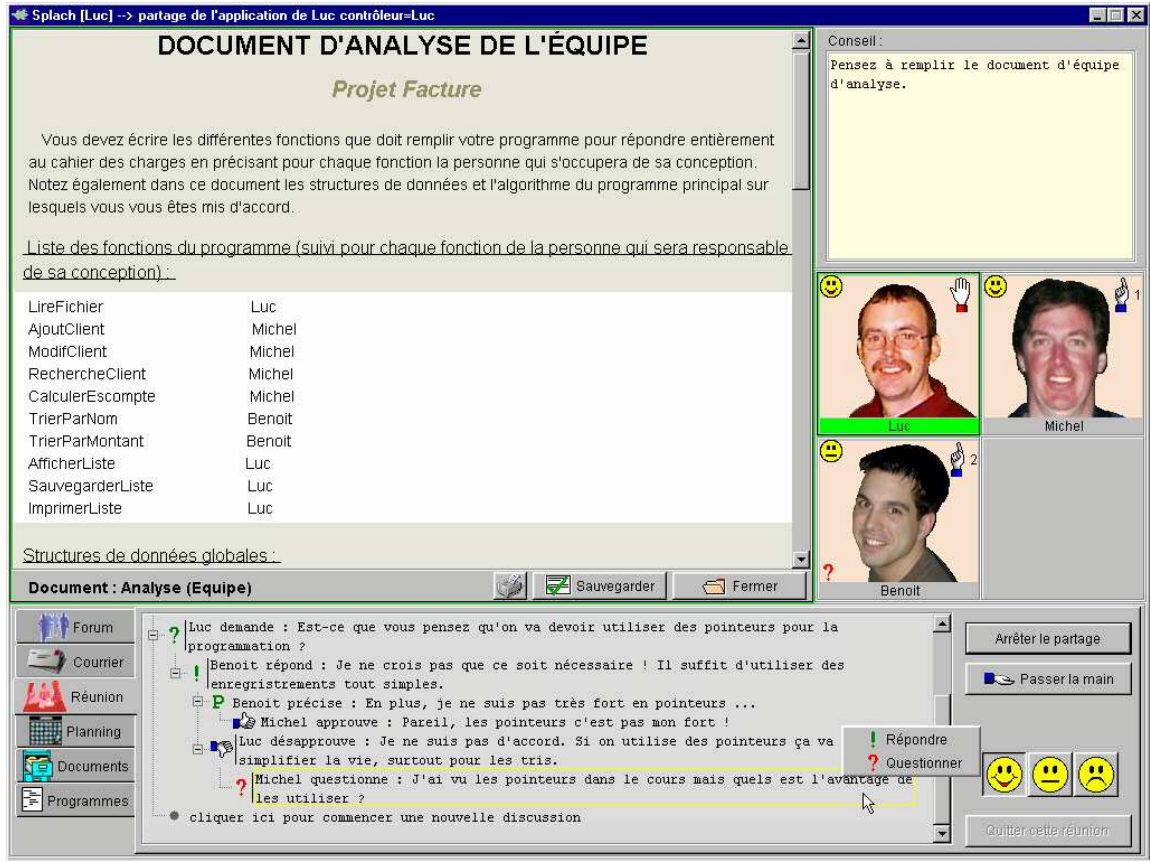

Figure 4. L'interface de l'environnement SPLACH pendant une réunion

Dans le domaine des interfaces personne-machine, certains chercheurs se sont intéressés au développement de la conscience mutuelle (awareness) entre personnes d'un groupe utilisant un collecticiel [DOU 92]. Ils soulignent que la visibilité des actions individuelles est fondamentale dans l'interaction et l'interprétation des actions des autres. Cette conscience mutuelle permet de structurer l'activité, d'éviter la duplication du travail, de faciliter la progression du groupe et de coordonner les activités entre elles. Notre environnement cherche à produire cette conscience mutuelle en affichant, lors des réunions, les photos des participants dans la partie droite de l'interface. Par ailleurs, des boutons représentant des " emoticônes », encore appelé smileys, permettent à chaque participant de signaler une émotion $(:):(:)$ qui s'affiche à côté de sa photo.

\section{Analyse automatique de conversation textuelle pour la détermination de profils de comportements sociaux}

Nous venons de présenter un environnement visant à supporter les apprenants lors de projets collectifs. Nous utilisons le terme de support car cet environnement dédié à une pédagogie de projet facilite et structure les activités des apprenants. Nous envisageons un deuxième type de support, celui-ci étant fourni par un système 
d'assistance automatique. Nous pouvons même parler d'une double assistance car, d'un côté, nous voulons aider les apprenants dans la réalisation du projet et dans l'apprentissage du travail en équipe et, d'un autre côté, nous souhaitons également assister le chef de projet dans son suivi des activités à la fois individuelles et collectives. Nous ne pouvons aborder les problèmes d'assistance sans traiter celui du traçage des activités des apprenants. Par conséquent, l'un de nos buts est d'avoir un système qui puisse recueillir et analyser les données issues des activités de projet. Nous présentons dans cette partie notre travail sur une analyse particulière pour déterminer des profils de comportements sociaux lors de conversations synchrones.

\subsection{Objectifs recherchés dans la détermination de profils de comportements sociaux}

Nous savons qu'à l'intérieur de groupes de personnes des comportements sociaux apparaissent (animateur, indépendant, etc.), y compris dans les groupes virtuels [RHE 93]. Dans un contexte éducatif, il est intéressant de chercher à déterminer automatiquement ces profils de comportements sociaux à l'intérieur des groupes d'apprenants car ils peuvent être utiles aussi bien pour le tuteur, pour les systèmes informatiques que pour les apprenants eux-mêmes. Pour le tuteur responsable de l'encadrement, des informations sur ces profils lui permettraient de mieux comprendre les relations sociales dans le groupe, de mieux suivre l'activité collective qui se déroule et donc de mieux encadrer les apprenants voire même d'utiliser ces profils comme une aide à la formation de futurs groupes d'apprenants. Pour les systèmes informatiques, une analyse automatique aboutissant à la détermination de profils comportementaux contribuerait à fournir des indicateurs pour des agents informatiques cherchant à apporter de l'assistance adaptée et des conseils personnalisés aux apprenants. En ce qui concerne les apprenants, leur fournir les informations sur leurs profils permettrait de leur donner une vue sur leurs comportements et leur donner ainsi un autre regard sur leur processus de travail. Nous venons de montrer les multiples intérêts d'une analyse automatique des profils de comportements sociaux dans un contexte de formation à distance. La question est de savoir comment parvenir à cette analyse automatique ? Nous présentons dans la suite de cette section l'état de nos travaux sur une approche pour y parvenir.

\subsection{Fondements pour l'analyse de comportements sociaux d'apprenants}

Robert Pléty, chercheur du laboratoire d'éthologie des communications de l'université Lumière-Lyon 2, a beaucoup étudié le comportement d'élèves travaillant en groupe; il a notamment analysé des interactions entre élèves ${ }^{4}$ travaillant en groupe de quatre à la résolution de problèmes d'algèbre [PLÉ 96]. Pour ce faire, il

\footnotetext{
${ }^{4}$ Les élèves de son étude étaient des collégiens en classe de 4ème.
} 
est parti d'une micro-analyse des échanges verbaux et gestuels dans le groupe pour aboutir à la détermination de profils de comportement chez les élèves.

Au sujet du travail d'élèves en groupe, Pléty précise qu'une organisation se met en place spontanément et rapidement, et qu' «au sein de cette organisation chacun des partenaires sait trouver, suivant ses capacités et ses connaissances personnelles, sa propre place » [PLÉ 96]. Pléty définit ainsi des profils de comportement qui caractérisent les rôles que les partenaires jouent dans le groupe. Ses analyses lui ont permis d'identifier des profils types de comportement. Afin de déterminer ces comportements quatre sortes d'observations sont effectuées pour chaque élève : le volume d'intervention, le type d'intervention, les gestes de type communicatif (regard et mouvement), les réactions des autres (ce que les interventions entraînent).

À partir de ces observations, Pléty fait ressortir quatre profils types de comportement: l'animateur, le vérificateur, le quêteur et l'indépendant. Les caractéristiques de ces quatre profils sont résumées dans le Tableau 1.

\begin{tabular}{|l|l|l|l|l|}
\hline Profil & \multicolumn{1}{|c|}{$\begin{array}{c}\text { Volume } \\
\text { d'intervention }\end{array}$} & \multicolumn{1}{c|}{$\begin{array}{c}\text { Type } \\
\text { d'intervention }\end{array}$} & $\begin{array}{c}\text { Gestes } \\
\text { communicatifs }\end{array}$ & \multicolumn{1}{c|}{$\begin{array}{c}\text { Réactions } \\
\text { entraînées }\end{array}$} \\
\hline Animateur & Important & $\begin{array}{l}\text { Interrogation ou } \\
\text { proposition }\end{array}$ & $\begin{array}{l}\text { Importants (regards } \\
\text { et mouvements) }\end{array}$ & $\begin{array}{l}\text { Suivi de réactions } \\
\text { positives }\end{array}$ \\
\hline Vérificateur & Assez important & $\begin{array}{l}\text { Réaction, réponse et } \\
\text { évaluation }\end{array}$ & $\begin{array}{l}\text { Régulateurs (regards } \\
\text { orientés) }\end{array}$ & $\begin{array}{l}\text { Peu suivi de } \\
\text { réactions }\end{array}$ \\
\hline Quêteur & Peu important & $\begin{array}{l}\text { Très dubitatif } \\
\text { questions) }\end{array}$ & $\begin{array}{l}\text { Regards longs de } \\
\text { quête }\end{array}$ & $\begin{array}{l}\text { Ses questions sont } \\
\text { bien acceptées }\end{array}$ \\
\hline Indépendant & Faible & $\begin{array}{l}\text { Pas ou peu de propo- } \\
\text { sition ni d'évaluation }\end{array}$ & Peu de regards & $\begin{array}{l}\text { Ses interventions } \\
\text { restent en suspens }\end{array}$ \\
\hline
\end{tabular}

Tableau 1. Les profils comportementaux d'élèves travaillant en groupe (synthèse personnelle d'après les travaux de Pléty)

D'après Pléty, il ressort de ces analyses que l'animateur et le vérificateur constituent l'axe fort des échanges. Un leadership peut être exercé par l'animateur et/ou par le vérificateur. Le quêteur et l'indépendant se positionnent alors en fonction des relations qui s'instaurent entre l'animateur et le vérificateur. Ainsi, les apprenants se définiraient «un rôle à l'intérieur du groupe de façon à régler au mieux les échanges et les rapports entre eux » [PLÉ 96].

Nous nous sommes alors posés la question de savoir si, à distance et avec les réseaux, il est possible de retrouver ces mêmes caractéristiques de groupes d'apprenants. Pléty apporte un élément de réponse, parlant de groupes d'apprenants distribués sur réseau, en soulignant que «curieusement, on y rencontre les mêmes aspects d'appartenance, de cohésion et de leadership que dans les groupes ordinaires » [PLÉ 98]. La détermination de profils de comportement pour des apprenants travaillant en groupe à distance semble donc avoir un sens.

Nous émettons donc l'hypothèse que les profils comportementaux relevés par Pléty peuvent se retrouver dans les groupes d'apprenants travaillant à distance. La 
question se pose de savoir comment transposer les travaux de Pléty dans un contexte de conversation médiatisée. Une des manières d'y arriver est d'analyser automatiquement les conversations entre apprenants.

\subsection{Analyse de conversation et actes de langage}

Pour notre travail, nous nous intéressons aux conversations médiatisées synchrones de forme textuelle. Une conversation peut être considérée comme une succession de tours de parole [SAC 74], c'est-à-dire une suite d'échanges composés d'interventions de locuteurs et constituées elles mêmes d'actes de langage dont l'un est l'acte directeur [ROU 85]. Si l'on cherche à analyser automatiquement une conversation, il est alors important de repérer ces actes de langage directeurs. À l'heure actuelle, une des techniques pour analyser les conversations consiste à fournir une interface semi-structurée pour demander un acte de langage à l'utilisateur avant que celui-ci ne saisisse le contenu de son message de manière libre (d'où le terme de semi-structuration).

Même si l'utilisation d'une interface semi-structurée par des actes de langage comporte des inconvénients, comme le manque de flexibilité ou l'ajout de contraintes et de discipline pour l'utilisateur [SUC 94], c'est une technique qui peut être utile dans un contexte d'apprentissage pour les raisons suivantes :

- le fait de typer un message amène l'utilisateur à se demander ce qu'il veut dire et a donc une valeur éducative [WIN 87 ; FLO 88] ;

- une interface semi-structurée encourage les utilisateurs à se centrer davantage sur la tâche [BAK 97 ; JER 97];

- l'utilisation d'ouvreurs permet de concevoir des systèmes d'analyse automatique de discussions [MCM 95].

Il nous semble donc parfaitement opportun d'utiliser une structuration au moyen d'actes de langage pour, d'une part, concevoir un outil de conversation dans un contexte éducatif et, d'autre part, analyser automatiquement des profils de comportements sociaux. Nous sommes conscients des critiques qui existent à ce sujet et nous tentons d'apporter certaines solutions dans la section suivante notamment par rapport aux points suivants :

- la structuration de l'environnement doit par elle-même encourager à utiliser l'acte de langage correspondant à l'intervention [ROB 98] ;

- un grand nombre d'actes rend l'interface difficilement utilisable [SOL 99] ;

- l'outil doit permettre de rendre le fil des conversations explicite [ROB 98].

\subsection{Choix des actes de langage}

Les actes que nous proposons d'intégrer dans un outil de conversation semistructuré se répartissent en cinq catégories. Trois de ces catégories se trouvent par 
exemple dans les travaux de [ROU 86] à savoir : les actes initiatifs, les actes réactifs et les actes évaluatifs. Nous avons ajouté à ces trois catégories de bases deux autres catégories. La première est une catégorie que nous avons nommée de «salutation » [KEN 73 ; LAV 81]. La deuxième catégorie ajoutée est celle des actes auto-réactifs. Nous n'avons pas trouvé de travaux y faisant référence. Nous pensons néanmoins qu'il est nécessaire de pouvoir réagir à ses propres interventions. Il est vrai que cette catégorie peut se confondre avec la catégorie des actes réactifs. Cependant, nous préférons distinguer clairement les interventions de réaction d'un locuteur à l'une de ses propres interventions, de celles réagissant à des interventions émises par d'autres. Le Tableau 2 précise les actes que nous avons identifiés pour chacune des catégories retenues.

\begin{tabular}{llc}
\hline Catégories & Actes de langage & Icônes \\
\hline Salutation & Saluer & \\
\hline Initiatif & Proposer & $?$ \\
& Demander & $?$ \\
& Affirmer & $!$ \\
\hline Réactif & Répondre & $?$ \\
& Questionner & Epprouver \\
\hline Évaluatif & Désapprouver & $\mathbf{P}$ \\
& Préciser & $\mathbf{R}$ \\
\hline Auto-réactif & Rectifier & \\
& &
\end{tabular}

Tableau 2. Les actes de langage proposés pour notre outil de conversation

Afin de faciliter le travail de sélection des actes de langage par l'utilisateur, nous avons cherché à définir un nombre assez restreint d'actes distants sémantiquement. Nous arrivons à dix actes au total. En comparant à d'autres taxinomies des actes de langage, il est vrai que nous n'avons pas une granularité aussi fine. Cependant nous ne cherchons pas ici à concevoir une taxinomie théorique. Nous cherchons avant tout à avoir un ensemble d'actes exploitables facilement par l'utilisateur et suffisant pour l'analyse que nous voulons en faire, c'est-à-dire pour la détermination des profils comportementaux. Des actes comme «demander» et «questionner» peuvent paraître identiques mais ils ne seront pas disponibles en même temps car ne faisant pas partie de la même catégorie. Enfin, il faut signaler que le Tableau 2 est le résultat d'une confrontation de notre outil de conversation avec les utilisateurs lors d'une première expérimentation de l'environnement SPLACH (cf. section 5.1).

Par ailleurs, dans une conversation, les actes de langage obéissent à des contraintes d'enchaînement. Nous adhérons particulièrement à l'idée de paires adjacentes [SCH 73 ; CLA 89]. Selon ces auteurs, une paire adjacente se compose 
de deux expressions ordonnées produites par deux locuteurs, la forme et le contenu de la deuxième partie de la paire étant dépendante de la première partie. Nous utilisons cette idée de paire adjacente pour atteindre deux de nos objectifs : faciliter la structuration des conversations et contribuer à limiter les sélections inappropriées d'actes de langage. La Figure 5 représente un graphe de successions des actes de langage présenté précédemment. Ce graphe est le résultat d'un processus itératif incluant des expérimentations auprès d'utilisateurs. Le nœud de gauche représente l'initiation d'une nouvelle discussion. Ainsi, un utilisateur peut commencer une discussion par une salutation («saluer») ou par un acte initiatif («demander», «proposer» ou «affirmer»). Pour prendre un autre exemple, à partir d'une proposition, un utilisateur peut «questionner» cette proposition ou l'évaluer («approuver» ou «désapprouver»). Pour les actes auto-réactifs, lorsqu'un utilisateur a fait une intervention, quel que soit le type, il peut réagir à sa propre intervention en précisant ou rectifiant ce qu'il vient de dire.

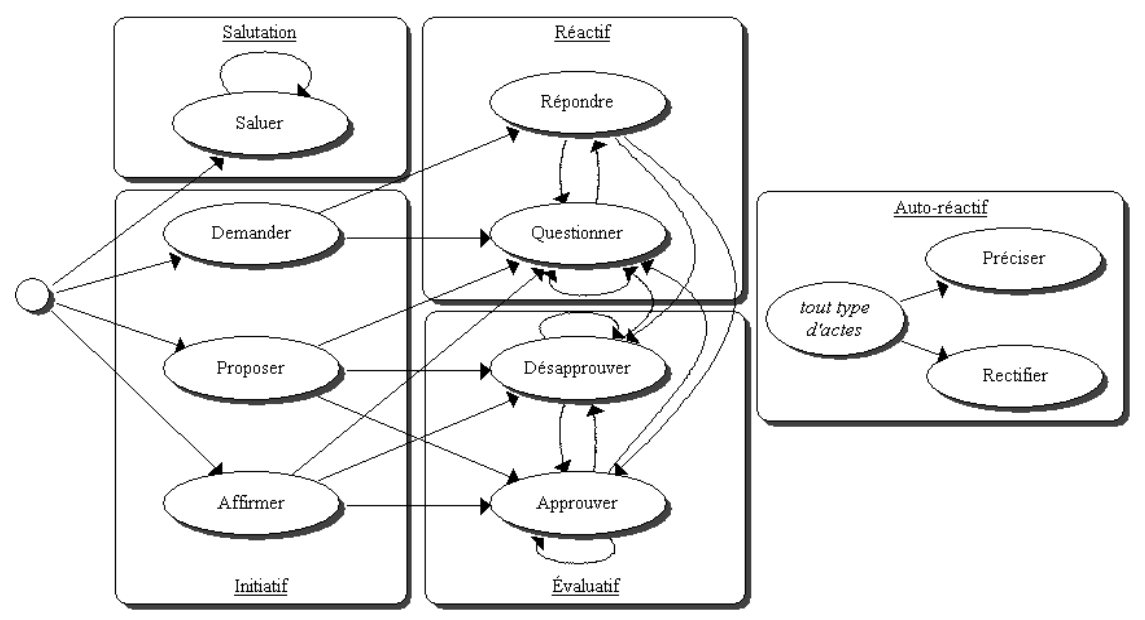

Figure 5. Graphe de successions des actes de langage

Nous pouvons par ailleurs remarquer que le graphe de successions ne présente pas de nœud de fin. Cette caractéristique vise à permettre aux débats de se dérouler. Cet aspect est important dans un contexte d'apprentissage pour favoriser l'approfondissement des idées (ici l'objectif n'est pas que les utilisateurs trouvent un accord le plus rapidement possible mais qu'ils puissent échanger et partager leurs connaissances).

\subsection{Représentation arborescente des messages}

Un grand nombre d'outils de communication écrite synchrone existe sur le marché (outils de chat). Cependant, ces outils n'ont pas beaucoup évolué depuis une 
vingtaine d'années et demeurent peu adaptés pour des conversations soutenues. Leur fonctionnement repose sur un empilement des messages des utilisateurs de façon temporelle. Nous formulons des critiques à ce fonctionnement. Cet empilement de messages les uns à la suite des autres selon leur ordre d'arrivée pose des problèmes dans un contexte de conversation synchrone. En effet, le temps nécessaire à la composition des textes (temps de frappe) ne permet pas de répondre de façon immédiate à un message. De ce fait, les temps de latence provoquent des imbrications des interventions qui rendent le suivi de la discussion difficile. Deux messages peuvent se retrouver proches à l'interface alors qu'ils ne sont pas forcément liés et, à l'inverse, deux messages en relation peuvent être séparés par d'autres messages. La conséquence de ce défaut majeur est d'avoir une conversation délicate à suivre et à mener. Des résultats d'analyse de discussions médiatisées par des outils de chats ont montré que celles-ci étaient très souvent incohérentes et qu'on trouvait de nombreux messages de « réparation » de la discussion [HER 99].

Afin de pallier ce problème d'imbrication, nous proposons de munir l'outil de conversation d'une représentation arborescente des messages. L'organisation des messages sous forme arborescente est beaucoup utilisée dans les forums de discussion asynchrones. Le principe est de lier chaque message à celui auquel il répond ou réagit. Les nouveaux sujets de discussion sont placés à la racine de l'arbre, les autres se raccrochant aux messages existants. L'avantage de cette représentation est de tenir compte des fils de discussion et donc des sujets de conversation. Peu de recherches ont été menées sur la représentation arborescente des conversations synchrones médiatisées. Depuis la création de notre outil, nous avons néanmoins trouvé un autre travail de recherche allant dans ce sens. Ainsi, un prototype d'interface nommée Threaded Chat a récemment été développé dans les laboratoires de recherche de $\mathrm{Microsoft}^{\odot}$ [SMI 00]. Une étude expérimentale de cet outil a permis de voir qu'il était prometteur et qu'il facilitait notamment l'aide à la prise de décision. Cependant, dans cet outil, chaque message ne s'affiche que sur une seule ligne ce qui oblige l'utilisateur à faire défiler le message horizontalement pour le lire dans sa totalité. L'outil que nous avons conçu (cf. Figure 4) n'a pas ce problème ergonomique grâce à un affichage des messages sur plusieurs lignes lorsque cela est nécessaire. Cette représentation arborescente des messages contribue à faciliter l'utilisation des actes de langages appropriés car elle permet de les proposer de manière contextuelle.

\subsection{Calcul des profils comportementaux}

À partir des spécifications précédentes concernant la conception d'un outil de conversation, il nous est possible de concevoir un système d'analyse automatique afin d'identifier les quatre profils de comportements types relevés par Pléty. Nous présentons ici les formules utilisées par un agent informatique que nous avons conçu pour calculer les profils comportementaux des utilisateurs pendant une conversation. Ces formules heuristiques ont été déterminées à partir des indications provenant des 
travaux de Pléty et ont été affinées lors d'expérimentations. Une première formule permet de calculer tout d'abord le coefficient de participation d'un participant $p$ en divisant le nombre de messages envoyés par celui-ci par le nombre moyen de messages ${ }^{5}$ envoyés par les participants. Nous avons donc la formule suivante :

$$
\operatorname{coeffParticipation}(p)=\frac{\text { nbreMessages }(p)}{\text { MoyMessages }} \times 50
$$

Si le nombre de messages envoyé par un participant est égal au nombre moyen de messages, alors le coefficient de participation de ce participant est de 50. Un coefficient de participation proche de 0 indique une faible participation alors qu'un coefficient proche de 100 indique une forte participation dans la conversation ${ }^{6}$.

Quatre formules permettent de calculer des coefficients correspondant aux quatre profils : coefficient d'animation, coefficient de vérification, coefficient de quête et coefficient d'indépendance. Par exemple, le coefficient d'animation du participant $p$ est calculé par la formule suivante :

$$
\operatorname{coeffAnimation}(p)=\frac{n b M e s s a g e \text { Initiatifs }(p)}{n b \text { MessagesTotal }(p)} \times \frac{n b \text { TotalActes }}{\text { nbActesInitiatifs }} \times 50
$$

Dans cette formule, nbMessagesInitiatifs $(p)$ correspond au nombre de messages de type initiatif (i.e. proposer, affirmer, demander) envoyés par le participant et nbMessagesTotal $(p)$ correspond au nombre total de messages envoyés par ce même participant. Le rapport entre ces deux termes est multiplié par le rapport entre le nombre d'actes au total (10) et le nombre d'actes de type initiatif possibles (3). Lorsque ce coefficient se rapproche de 0 , cela signifie que le participant n'est pas animateur alors qu'un coefficient proche de 100 signale un fort comportement d'animation. Les calculs des autres coefficients sont obtenus de manière similaire.

Dès lors, les profils comportementaux sont calculés en pondérant le coefficient quantitatif de participation avec un des quatre coefficients plus qualitatifs décrits cidessus. Un coefficient de pondération ${ }^{7}$ est appliqué pour obtenir des valeurs représentatives et pertinentes. Ainsi, le profil d'animateur du participant $p$ est calculé par la formule suivante :

$$
\operatorname{profilAnimateur}(p)=\frac{\operatorname{coeffParticipation}(p)+2 \times \operatorname{coeffAnimation}(p)}{3}
$$

Dans ce cas, une pondération de $2 / 3$ accorde plus d'importance au coefficient d'animation par rapport au coefficient de participation. Les quatre profils sont calculés au fur et à mesure d'une conversation et évoluent au cours du temps. Nous présentons dans la section 5 un outil permettant d'observer l'évolution de ces profils.

\footnotetext{
${ }^{5}$ Cette moyenne tient compte de la présence du participant pour ne pas fausser le calcul si un participant arrive en cours de réunion. On ajoute $1 /$ nbParticipants à la moyenne à chaque message reçu. Cette moyenne n'est donc pas forcément la même pour tous les participants.

${ }^{6}$ Pour tous nos calculs, si un coefficient dépasse 100, il est alors ramené à 100.

${ }^{7}$ Ces coefficients de pondération ont été déterminés suite à des expérimentations.
} 


\section{Expérimentations de l'environnement SPLACH}

Une de nos préoccupations était de ne pas développer un environnement dédié à un domaine précis mais de réaliser un environnement qui puisse supporter des projets dans des domaines éducatifs divers. Afin de montrer cette qualité de « réutilisabilité », nous avons expérimenté SPLACH dans deux contextes différents ${ }^{8}$.

\subsection{Contextes d'expérimentations}

Une première expérimentation de SPLACH a été réalisée avec quinze élèves en classe de 4ème et de 3ème (13-14 ans) dans le domaine de la robotique pédagogique. L'objectif des projets était de construire des robots pour participer à un concours' Les élèves ont travaillé pendant 3 mois à distance - définition, partage et réalisation de différents modules de robots - avant de se rencontrer pour assembler les différents modules. Une deuxième expérimentation de SPLACH a été réalisée dans un contexte de téléformation à la Télé-université du Québec. Six étudiants suivant des cours de programmation à distance ont formé deux équipes pendant six semaines. L'objectif global était de réaliser un programme en Pascal de manière collective, chaque étudiant étant responsable de fonctions du programme.

Pour les deux expérimentations, l'objectif initial des projets a été atteint : construction et programmation d'un robot pour les élèves de collège, écriture d'un programme pour les étudiants de la Télé-université. L'observation des activités a montré que les productions finales ont été le fruit d'un réel travail collectif. Ces expérimentations nous ont ainsi permis de valider l'environnement SPLACH d'un point de vue technique et fonctionnel, en montrant qu'il peut effectivement soutenir une pédagogie de projet à distance. Nous donnons dans les paragraphes suivants des résultats ${ }^{8}$ issus de ces expérimentations en nous centrant sur l'usage des documents de projet et sur l'analyse automatique des profils comportementaux. Ces résultats sont principalement fondés sur les observations que nous avons pu faire et sur une analyse de questionnaires remplis par les apprenants.

\subsection{Utilisation des documents de projet}

Pour la première expérimentation en collège, nous avions anticipé les problèmes que pouvait poser la conception d'un robot commun à distance en donnant la possibilité aux élèves d'insérer des schémas ou des photos de leurs robots dans leurs documents. Cette fonctionnalité a été utilisée par la plupart des élèves et s'est révélée utile à la coopération. Par exemple, un groupe d'élèves a prévenu un autre groupe,

\footnotetext{
${ }^{8}$ Une description plus détaillée des expérimentations se trouve dans [GEO 01].

${ }^{9}$ L'objectif du concours était de concevoir des robots devant suivre une ligne noire tout en réalisant diverses actions : faire tomber une quille, mettre une balle dans un trou, etc.
} 
en voyant la photo de leur robot, que trois roues ne suffisaient pas et qu'une quatrième était nécessaire pour la stabilité du robot. Dans ce sens, les documents sont utiles pour l'anticipation de l'intégration des différents travaux. Les gabarits des documents individuels de projet ont bien été remplis par les élèves jusqu'à la phase de construction. Après, les élèves ont passé plus de temps à mettre au point leur robot qu'à rédiger les documents. Les informations passaient alors davantage par les communications asynchrones et synchrones. Par contre, si ce n'est lors de la première réunion d'analyse du projet, les documents d'équipe n'ont pas été utilisés par les apprenants. Les réunions synchrones qui avaient lieu une fois par semaine servaient davantage à la discussion qu'à la co-conception d'un document. Néanmoins, les documents d'équipe ont été utilisés par les chefs de projet pour synthétiser les décisions prises lors des communications asynchrones ou synchrones.

Pour la deuxième expérimentation, les étudiants ont été amenés à effectuer deux types de production : des documents, en remplissant les gabarits proposés (analyse, algorithmes) et des programmes en Pascal. Les étudiants ont respecté la rédaction des documents telle qu'elle était définie dans la structuration des projets. Ainsi, ils pouvaient voir l'évolution des différentes productions de chacun. Avec cette information partagée, les étudiants ont pu détecter des conflits et les résoudre ensemble. La coordination a pu se faire grâce à la visibilité des activités individuelles dans le travail collectif. D’une manière générale, nous avons remarqué que les conversations synchrones durent en moyenne une heure. En effet, le synchrone est possible mais contraignant (il faut trouver un horaire commun, il faut que l'ordinateur et la ligne téléphonique soient disponibles pendant le temps de la réunion). Une heure passe très vite lors d'une conversation textuelle synchrone et il est alors difficile d'écrire un document en collaboration dans ce laps de temps. La plupart des documents à réaliser collectivement ont été faits par un copier coller de l'un des participants suivi d'une validation par les autres.

Pour résumer les résultats des deux expérimentations, nous avons constaté que les documents étaient utiles pour la coordination du travail collectif. De plus, les gabarits facilitent la rédaction de ces documents. Cependant, la co-conception synchrone de document a été peu utilisée et, lorqu'elle l'a été, c'était davantage le travail d'un apprenant que les autres validaient.

\subsection{Résultats sur la détermination automatique de comportements sociaux}

L'analyse automatique des conversations synchrones a été mise en œuvre seulement lors de la deuxième expérimentation. La première expérimentation a quant à elle permis de déterminer les actes de langages de l'interface semi-structurée. Les étudiants de notre deuxième expérimentation ont apprécié l'outil de conversation synchrone et n'ont pas trouvé gênant le fait de sélectionner un acte de langage même s'ils n'y ont pas toujours vu une utilité immédiate. Une analyse manuelle de l'utilisation des actes de langage nous montre que les étudiants les ont très souvent 
utilisés à bon escient (seulement 10 à $15 \%$ des actes sélectionnés ne correspondent pas au contenu du message). La sélection des actes par menu déroulant contextuel, issue de la mise en œuvre du graphe de succession, paraît pertinente.

L'expérimentation a permis de montrer que les profils comportementaux calculés étaient fidèles à ce qui se passait dans les conversations. Les formules de calculs élaborés semblent donc pertinentes par rapport à notre contexte d'usage. La Figure 6 montre un outil qui permet de visualiser l'évolution des profils comportementaux au cours d'une conversation. Cet outil a pour l'instant été conçu dans un objectif d'observation expérimentale mais il pourrait être fourni au tuteur pour l'assister dans le suivi des apprenants ou aux apprenants eux-mêmes pour leur donner une vue sur leurs propres comportements. Ainsi, après avoir vu a posteriori les résultats de cet outil, le chef de projet qui encadrait les étudiants a pensé qu'il lui aurait été utile pour avoir une vue sur les réunions auxquelles il n'avait pas assistées.

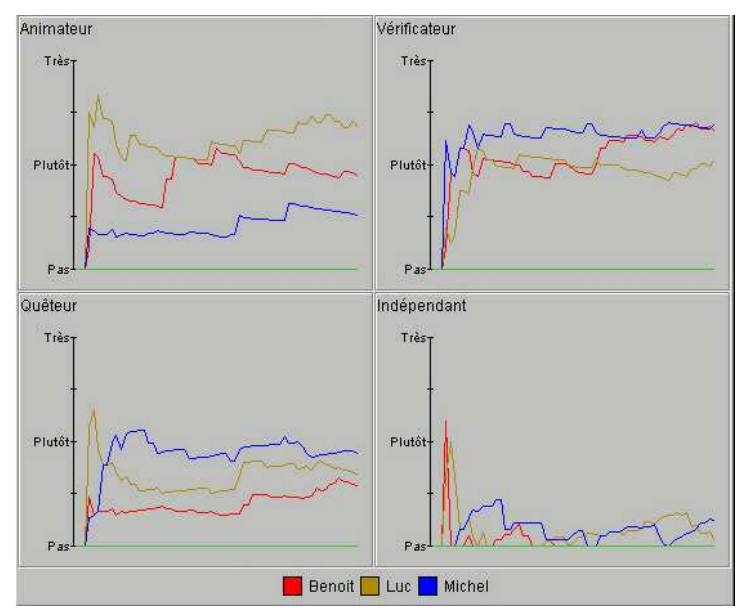

Figure 6. : L'outil de visualisation des profils comportementaux

Chaque graphe correspond à un type de profil particulier et les courbes représentent l'évolution dans le temps des profils des utilisateurs présents à la réunion. Ainsi, le graphe «Animateur » de la Figure 6 signale que l'utilisateur Luc a majoritairement tenu un rôle d'animateur lors de cette réunion alors que Michel a été le «moins animateur». Par ailleurs, nous pouvons voir que Michel a été le plus « quêteur » et qu'aucun participant n'a vraiment été « indépendant ».

\section{Conclusion et perspectives}

Nous avons présenté dans cet article nos travaux sur l'utilisation de documents et d'espaces de communication numériques dans le cadre d'un environnement CSCL 
support d'une pédagogie de projet. Nous espérons avoir démontré le rôle central que jouent ces documents et espaces comme supports aux activités collectives des apprenants tant au niveau structurel pour faciliter l'échange et le partage de connaissances qu'au niveau communication au sein d'une équipe.

Au niveau des documents numériques, nos travaux vont s'orienter vers un outil d'édition de gabarits de documents pour permettre la mise en place de projets par un non informaticien. Pour l'instant, l'édition des gabarits se fait en programmant en java. En fait, le programme est très simple et consiste uniquement à ajouter différents composants textes dans un document en précisant les attributs de chaque composant, en particulier modifiable ou non. L'exécution de ce programme génère alors le gabarit. La réalisation d'un éditeur de gabarit approprié ne présente pas de difficulté particulière et contribuera à une utilisation plus large de l'environnement SPLACH.

Les premiers résultats obtenus lors de l'expérimentation à la Télé-université, semblant confirmer notre hypothèse de retrouver les profils comportementaux relevés par Pléty dans les groupes d'apprenants travaillant à distance, nous encourage à poursuivre dans cette voie. Nous sommes conscients que les calculs pourraient être affinés en prenant d'autres variables en compte et en réalisant des expérimentations plus spécifiques. Dans un souci de simplification volontaire, nous ne prenons pas en considération les réactions aux interventions dans les calculs (par exemple une intervention d'un animateur se caractérise aussi par le fait qu'elle entraine souvent d'autres réactions). Notre volonté est d'avoir des calculs peu complexes pour le moment et ne mettant pas en jeu un trop grand nombre de paramètres. Néanmoins, dans notre méthode incrémentale de conception, nous gardons cette idée de prendre en compte les réactions aux interventions en perspective afin d'affiner les calculs. Une autre perspective à nos travaux concerne l'approfondissement de l'étude sur l'outil de visualisation de profils de comportementaux et d'une façon plus large l'instrumentation du formateur dans son rôle d'encadrement des apprenants dans un contexte d'apprentissage collectif.

\section{Remerciements}

Cette recherche a été en partie réalisée dans le cadre du collectif scientifique LÉA qui est soutenu par la commission permanente de coopération francoquébecoise. Nous tenons également à remercier toutes les personnes qui ont permis de réaliser nos expérimentations en situation réelle.

\section{Bibliographie}

[BAK 97] Baker M. J., Lund K., "Promoting reflective interactions in a computer-supported collaborative learning environment", Journal of Computer Assisted Learning, vol. 13, 1997, p. 175-193.

[BAN 89] Bannon L. J., "Issues in Computer-Supported Collaborative Learning", C. O'Malley (Ed.), NATO Advanced Workshop on Computer-Supported Collaborative Learning, Maratea, Italy, Sep. 1989. 
[BÉD 98] Bédard D., Bélisle M., "Contribution des technologies de l'information et de la communication (TIC) au développement des compétences discursives", Revue DFLM, vol. 2, 1998, p. 35-39.

[CAP 68] Caplow T., Deux contre un. Les coalitions dans les triades, Collection sciences humaines appliquées, Les éditions ESF, 1968.

[CER 99] Cerratto T., "Activités collaboratives sur réseau, une approche instrumentale de l'écriture en collaboration", Thèse de Doctorat en Psychologie cognitive, Université Paris 8, 1999.

[CLA 89] Clark H. H., Schaefer E. F., "Contributing to Discourse", Cognitive Science, vol. 13, 1989, p. 259-294.

[DIR 94] Dirckinck-Holmfeld L., "Project Pedagogy as the Foundation for Computer Supported Collaborative Learning", Joint Working Conference, Nantes, France, 27-30 octobre 1994.

[DOU 92] Dourish P., Bellotti V., "Awareness and Coordination in Shared Work Spaces", ACM Conference on Computer-Supported Cooperative Work CSCW'92, Toronto, Canada, novembre 1992, p. 107-122.

[FIS 00] Fischer G., "Shared Understanding, Informed Participation, and Social Creativity Objectives for the Next Generation of Collaborative Systems", R. Dieng, A. Giboin, L. Karsenty, G. De Michelis (Eds.), Fourth International Conference on the Design of Cooperative Systems, COOP'2000, Sophia Antipolis, France, 23-26 mai 2000, IOS Press, p. 3-16.

[FLO 88] Flores F., Graves M., Hartfield B., Winograd T., "Computer Systems and the Design of Organizational Interaction", ACM Transactions on Office Information Systems, vol. 6, nº 2,1988 , p. $153-172$.

[GEO 01] George S., "Apprentissage collectif à distance. SPLACH : un environnement informatique support d'une pédagogie de projet", Thèse de Doctorat en Informatique, Université du Maine, 2001.

[GOG 94] Goguelin P., "Comment faire naître un projet", Dossier Projet, Sciences Humaines, vol. 39, nºmai, 1994, p. 30-31.

[HER 99] Herring S., "Interactional Coherence in CMC", Journal of Computer-Mediated Communication, vol. 4, n4, 1999, p. http://www.ascusc.org/jcmc/vol4/issue4/.

[JER 97] Jermann P., Schneider D., "Semi-structured interface in collaborative problemsolving", First Swiss Workshop on distributed and parallel systems.

[KEN 73] Kendon A., Ferber A., A description of some human greetings, in Comparative ecology and behaviour of primates, R. P. Michael, J. H. Crook, Eds. (Academic Press, London, 1973) p. 591-668.

[LAV 81] Laver J., Linguistic routines and politeness: Greeting and parting, in Conversational routines, F. Coulmas, Ed. (Mounton, New York, 1981) p. 289-305.

[MCM 95] McManus M. M., Aiken R. M., "Using an Intelligent Tutor to Facilitate Collaborative Learning", Innovating Adult Learning with Innovative Technologies. Elsevier Science B.V., 1995, p. 49-64. 
[PAQ 96] Paquette G., "La modélisation par objets typés - une méthode de représentation pour les systèmes d'apprentissage et d'aide à la tâche", Sciences et Techniques Éducatives, vol. $3, \mathrm{n}^{\circ} 1,1996$, p. $9-42$.

[PLÉ 96] Pléty R., L'apprentissage coopérant, Ethologie et psychologie des communications, ARCI Presse Universitaire, Lyon, 1996.

[PLÉ 98] Pléty R., Comment apprendre et se former en groupe, Retz, 1998.

[POR 98] Portine H., "L'autonomie de l'apprenant en questions", Apprentissage des Langues et Systèmes d'Information et de Communication, vol. 1, n¹, 1998, p. 73-77.

[RHE 93] Rheingold H., Les communautés virtuelles., Addison-Wesley France, Paris, 1993.

[ROB 98] Robertson J., Good J., Pain H., "BetterBlether: The Design and Evaluation of a Discussion Tool for Education", International Journal of Artificial Intelligence in Education, vol. 9, 1998, p. 219-236.

[ROU 85] Roulet E., Auchlin A., Moeschler J., Schelling M., Rubattel C., L'articulation du discours en français contemporain, Collection Sciences pour la communication, Peter Lang, Berne, 1985.

[ROU 86] Roulet E., "Complétude interactive et mouvements discursifs", Cahier de Linguistique Française, vol. 7, 1986, p. 189-206.

[SAC 74] Sacks H., Schegloff E. A., Jefferson G., "A Simplest Systematics for the Organization of Turn-Taking for Conversation", Language, vol. 50, n4, 1974, p. 696-735.

[SCH 73] Schegloff E. A., Sacks H., "Opening Up Closings", Semiotica, vol. 8, n²4, 1973, p. 289-327.

[SIM 02] Simmel G., "The number of members as determining the sociological form of the group", American Journal of Sociology, vol. 8, n¹, 1902, p. 1-46.

[SMI 00] Smith M., Cadiz J. J., Burkhalter B., "Conversation Trees and Threaded Chats", Proceedings of the 2000 ACM Conference on Computer Supported Cooperative Work, Philadelphia, Pennsylvania, USA, 2-6 december 2000, p. 97-105.

[SOL 99] Soller A., Linton F., Goodman B., Lesgold A., "Toward Intelligent Analysis and Support of Collaborative Learning Interaction", S. P. Lajoie, M. Vivet (Eds.), Ninth International Conference on Artificial Intelligence in Education, Le Mans, 1999, IOS Press, p. $75-82$.

[SOU 96] Soubie J.-L., Buratto F., Chabaud C., La conception de la coopération et la coopération dans la conception, in Coopération et Conception, G. de Terssac, E. Friedberg, Eds. (Octares Editions, 1996) p. 187-206.

[SUC 94] Suchman L. A., Do Categories Have Politics? - The languagelaction perspective reconsidered, in Computer-Supported Cooperative Work, . (Kluwer Academic Publishers, 1994), vol. 2, p. 177-190.

[WIN 87] Winograd T., "A Language/Action Perspective on the Design of Cooperative Work", Human-Computer Interaction, vol. 3, n¹, 1987, p. 3-30. 